\title{
Objets et relations spatiales composites et prise en compte du vague pour interpréter un référencement spatial indirect
}

\author{
Mattia Bunel $^{1}$, Ana-Maria Olteanu-Raimond ${ }^{1}$, Cécile Duchêne ${ }^{1}$ \\ ${ }^{1}$ Univ. Paris-Est, LASTIG MEIG, IGN, ENSG, F-94160 Saint-Mandé, France \\ prenom.nom@ign.fr
}

RESUME. Cet article propose une méthodologie destinée à transformer une description de position exprimée dans un référentiel indirect en une position absolue dans un référentiel direct. Cette problématique nécessite une formalisation des éléments de localisation relatifs, ici sous la forme d'un modèle en triplets, utilisé ensuite comme base de référencement, et un questionnement sur la prise en compte de l'imperfection du langage naturel dans le processus de modélisation. Nous proposons de faire appel aux objets spatiaux flous et nous comparerons leurs implémentations afin d'identifier l'approche la plus adaptée au contexte du secours en montagne.

ABSTRACT. This paper proposes a methodology for transforming a position description expressed relatively in an indirect spatial reference system into an absolute position in a direct spatial reference system. This problem requires (1) to formalize the relative location elements, here in the form of a triplet model, which is used as a basis for the transformation from relative to absolute position, and (2) to investigate how the imperfection of the natural language can be taken into account in the modeling process. We propose to use fuzzy spatial objects and we will compare their implementations to identify the most appropriate approach to our context.

MotS-CLES: Référentiel spatial indirect, logique floue, objets spatiaux vagues, relation spatiale étendue, secours en montagne

KEYWORDS: Indirect spatial referential, fuzzy logic, ague spatial objects, extended spatial relation, mountain rescue

DOI:10.3166/RCMA.25.1-n @ 2019 Lavoisier 


\section{Introduction}

Lors de leurs interventions, les Pelotons de Gendarmerie de Haute Montagne (PGHM) peuvent être contraints à identifier manuellement la position d'une victime en recoupant des informations obtenues par téléphone (e.g. itinéraire emprunté, description verbale de position) avec des données métier, d'origines et de formats multiples (e.g. cartes numériques, guides papier). La complexité de cet exercice en fait le principal goulot d'étranglement de la phase de préparation des secours, conduisant généralement à la perte d'un temps précieux. L'ANR CHOUCAS ${ }^{1}$ vise à répondre à un besoin exprimé par le PGHM de Grenoble, en proposant des méthodes facilitant le processus d'identification de la position des victimes. Cela passe notamment par des travaux sur la constitution et l'enrichissement de données provenant de sources hétérogènes, ainsi que sur le raisonnement spatial et la visualisation interactive pour l'aide à la décision (Olteanu-Raimond et al., 2017).

$\mathrm{Au}$ sein de ce projet, notre travail part du constat que la victime décrit systématiquement sa position au moyen de relations spatiales et d'objets géographiques servant de référence, i.e. à l'aide d'un référentiel spatial indirect. Nous traiterons ici de leur transformation en des positions exprimées dans un référentiel direct (Clementini, 2011) compte tenu des spécificités de notre contexte. Au-delà de l'application au secours en montagne, cette problématique a pour enjeu un accès facilité à l'information géographique, plus proche de la cognition humaine que ce que permettent les outils et méthodes classiques disponibles sur SIG, comme prôné par Egenhofer et Mark (1995) avec la naive geography.

Cet article présentera la question de la modélisation d'une position à partir d'un référentiel indirect (section 2), ainsi que les principes de modélisation des relations spatiales retenus (section 3). La section 4 abordera la nécessité d'utiliser des objets spatiaux vagues et présentera un état de l'art des modèles et implémentations existantes, avant d'en confronter deux d'entre elles à un cas réel (section 5). La section 6 abordera la question des objets de référence composites. Nous présenterons ensuite une analyse de sensibilité des paramètres utilisés lors de nos modélisations (section 7), avant de conclure.

Ce papier est une version étendue d'un papier présenté à la conférence SAGEO (Bunel et al. 2018). La section 3 a été enrichie pour aborder le cas des objets de référence composites, les sections 6 et 7 ont été ajoutées et quelques modifications ont été apportées au reste du document.

\section{Modélisation d'un élément de localisation dans un référentiel indirect}

Dans cette partie, nous aborderons la question de la modélisation formelle d'un objet spatial à partir d'un référentiel indirect.

\footnotetext{
${ }^{1}$ ANR CHOUCAS : Intégration de données hétérogènes et raisonnement spatial pour l'aide à la localisation des victimes en montagne. ANR-16-CE23-0018
} 
Objets et relations spatiales composites pour interpréter un référencement spatial indirect. 3

\subsection{Hypothèses de travail}

Le modèle que nous présentons est fondée sur l'hypothèse que le requérant (personne contactant les secours) décrit une position à l'aide d'une ou plusieurs phrases telles que "Je suis à côté d'un arbre et en face d'un chalet » ou «Le blessé est à côté de moi ».

Notons que nous ne travaillons pas directement à partir de phrases mais avec une liste d'éléments de localisation, (e.g. «Je suis à côté d'un arbre » et «Je suis en face d'un chalet») extraits du discours du requérant par le secouriste et saisis au travers d'une interface également développée au sein de l'ANR CHOUCAS (dont il ne sera pas question ici).

La méthodologie que nous travaillons à définir s'articule en deux phases complémentaires, la première visant à identifier l'ensemble des positions correspondant à chaque élément de localisation donné par le requérant et la seconde consistant en l'agrégation de ces positions en vue d'identifier les positions correspondant à l'ensemble des éléments de localisation. L'imprécision est prise en compte durant tout le processus, ce n'est cependant pas le cas de l'incertitude, qui n'est modélisée qu'à partir de la seconde étape, la même incertitude caractérisant alors toutes les positions décrites par un même élément de localisation. Nous nous concentrons ici sur la première de ces phases, c'est-à-dire à la modélisation de l'imprécision des éléments de localisation.

\subsection{Formalisation des éléments de localisation}

Les éléments de localisation présentent une structure commune, composée d'un sujet dont on décrit la localisation ( $j e »$, «le blessé »), d'un point de repère défini comme l'objet de référence ("un arbre », «un chalet», «moi ») et une relation indiquant la position du sujet par rapport à l'objet de référence, que nous nommerons la relation spatiale («à côté », « en face »). D'autres travaux nomment différemment le sujet et l'objet de référence, par exemple Vandeloise (1986, p.20) les appelle respectivement cible et site. De manière analogue au modèle de Vasardini et al. (2013), nous proposons de modéliser ces éléments de localisation par un triplet composé de ces trois éléments. Ainsi les exemples précédents peuvent êtres modélisés par les triplets suivants : (je, à côté, arbre), (je, en face, rivière) ; (le blessé, à côté, moi).

Cependant une phrase telle que : "je suis presque en dessous d'un télésiège » n'est pas efficacement modélisée à l'aide d'une relation spatiale, la nuance apportée par l'adverbe presque étant ignorée. De manière analogue au modèle des entités nommées étendues ( expanded named entity») de Moncla et al. (2015), nous proposons de définir une relation spatiale étendue englobant la relation spatiale, ses modifieurs (e.g. presque, complétement, [à] $50 \mathrm{~m}$ ) mais également le verbe, ce dernier pouvant modifier le propos (e.g. se trouver vs voir). La composition de la relation spatiale étendue peut varier en fonction du contexte, mais elle est toujours composée d'au moins un verbe et d'une relation spatiale, les modifieurs étant 
facultatifs et illimités. Un élément de localisation peut alors être modélisé avec un triplet $E$ de la forme :

$$
E=(S, R S E, O) \text { avec } R S E=(V, \bmod , R)
$$

Où $S$ est le sujet, $R S E$ une relation spatiale étendue, $O$ l'objet de référence, $V$ le verbe, $R$ une relation spatiale et $\bmod$, le ou les modifieurs.

\section{Principes de modélisation des relations spatiales étendues}

Un des principaux verrous à la construction d'objets spatiaux à partir d'un référentiel indirect est l'interprétation des éléments de localisation. Bien que notre travail soit facilité par l'action du secouriste, nous restons confrontés à l'implicite et à la polysémie du langage humain. Cela pose deux principaux problèmes que nous proposons de résoudre à l'aide de trois principes de modélisation des relations spatiales étendues : la décomposition des relations, des objets de référence et la nonbivalence des relations.

\subsection{Décomposition des relations spatiales étendues}

Une même relation spatiale (et par extension une relation spatiale étendue), peut avoir des significations différentes en fonction de la nature de l'objet de référence (Borillio, 1998, pp. 16-17). Prenons l'exemple de la relation spatiale sous. Bien qu'ayant une sémantique proche, son sens dans les phrases «je suis sous un arbre » et «je suis sous une route», diffère. La notion de recouvrement présente dans la première phrase est absente de la seconde. Cette différence sémantique entre deux occurrences d'une même relation spatiale s'explique par la nature des objets de référence. Une personne peut être recouverte par un arbre et non par une route (à l'inverse d'un pont). Cela impose de prendre en compte les différentes sémantiques d'une même relation spatiale et ce en fonction des types d'objets de référence et des sujets utilisables dans ce contexte. Ces deux relations spatiales partagent toutefois une base sémantique commune : leur sujet est situé à une altitude inférieure à celle de l'objet de référence. Ce sont ces régularités sémantiques que nous proposons d'exploiter.

Notons $P a_{o}$ l'ensemble des positions situées à une altitude inférieure à un objet de référence de type $o$ :

$$
P a_{o}=\mathrm{U}\left(P a_{i} \mid i \in o\right)
$$

Où $o$ est l'ensemble des objets spatiaux d'un même type, $i$ une instance de $o$ et $P a_{i}$ l'ensemble des positions situées à une altitude inférieure à celle de $i$.

On peut alors construire les ensembles $P a_{r}$ et $P a_{a b}$ contenant les positions situées respectivement à une altitude inférieure à au moins une instance d'une route ou d'un arbre. Cependant, toutes les positions appartenant à $P a_{r}$ ou $P a_{a b}$ ne valident pas nécessairement les éléments de localisation «je suis sous une route » ou «je 
Objets et relations spatiales composites pour interpréter un référencement spatial indirect. 5

suis sous un arbre ». Ces ensembles contiennent des postions situées à une grande distance de l'objet de référence qu'il serait absurde de considérer comme situées sous l'un de ces objets de référence.

Dès lors, il apparait nécessaire de circonscrire la distance à l'objet de référence. Cependant (contrairement aux ensembles $P a_{r}$ et $P a_{a b}$ ) il n'est pas pertinent de fixer un même seuil de distance pour des objets de nature différente. La distance à partir de laquelle on ne se situe plus sous un arbre est en effet intuitivement plus faible que celle à partir de laquelle on ne se situe plus sous une route. Notons $P b_{o}$ l'ensemble des positions proches d'un objet de référence de type $o$. Nous pouvons alors construire une approximation des zones $P c_{r}$ et $P c_{a b}$, respectivement définies comme l'intersection des ensembles $P a_{r}$ et $P b_{r}$ ou $P a_{a b}$ et $P b_{a b}$.

Nous proposons donc de prendre en compte la polysémie des relations spatiales en les modélisant comme une composition de relations spatiales à la sémantique invariable et pondérées contextuellement, que nous nommons les relations spatiales atomiques. Nous supposons que ce travail de décomposition peut s'appliquer à toute relation spatiale non atomique. À titre d'exemple, la relation « entouré de » peut-être modélisée comme la conjonction des relations «à gauche », "à droite », « devant» et «derrière »(Vanegas et al., 2011). La principale difficulté de cette approche est qu'elle nécessite au préalable d'identifier les relations spatiales atomiques, ce qui impose une analyse manuelle des relations spatiales utilisées dans notre contexte.

\subsection{Objets de référence composites}

Dans certains cas, un objet de référence peut prendre la forme d'un regroupement d'objets spatiaux pouvant également faire office d'objets de référence. Le terme "télécabine », par exemple, peut aussi bien être utilisé pour qualifier les gares, que le câble porteur, les pylônes et par extension, l'union de ces éléments. Sans autres précisions, il est impossible de savoir à laquelle de ces composantes le mot «télécabine » fait référence.

Pour spatialiser un élément de localisation contenant un objet de référence composite, nous proposons d'ajouter au principe de décomposition des relations spatiales, un second principe de décomposition, portant sur les objets de référence. Cette décomposition méréologique consiste à identifier les différentes composantes de l'objet de référence. Ces dernières peuvent ensuite être spatialisées indépendamment, comme s'il s'agissait de deux éléments de localisation partageant le sujet et la relation spatiale. L'union des ensembles de positions permet alors de construire l'ensemble des positions correspondant à l'élément de localisation non décomposé.

Notons Po l'ensemble des positions correspondant à un élément de localisation dont l'objet de référence $o$ est composite. On peut exprimer $P o$ de la manière suivante :

$$
P o=U(P c \mid c \in o)
$$


Avec $c$, une composante de l'objet de référence et $P c$ l'ensemble des positions correspondant à l'élément de localisation ayant pour objet de référence $c$.

L'application de ce second principe nécessite cependant une connaissance des objets de référence utilisés dans le contexte du secours en montagne et des liens de composition qui peuvent exister entre eux.

\subsection{Non-bivalence des relations spatiales étendues}

Le principe de la non-bivalence des relations spatiales étendues consiste à considérer qu'il n'existe pas nécessairement deux espaces distincts dans lesquels on peut respectivement considérer que la relation est, et n'est pas, vérifiée. Ce principe découle de la nature imprécise du langage et de la difficulté de construire des objets spatiaux à partir de relations spatiales telles que «à côté » ou «sous». La construction des ensembles $P c_{r}$ et $P c_{a b}$ (section 3.1) nécessite la définition de seuils : un premier seuil représentant la différence d'altitude à partir de laquelle une personne n'est plus située sous un objet de référence, et deux seuils de distance : le premier représentant la distance à partir de laquelle on considère qu'une personne n'est plus sous un arbre, le second la distance à partir de laquelle on considère qu'une personne n'est plus sous une route.

Fixer précisément ces seuils est une opération délicate, car il est nécessaire de prendre en compte la nature de l'objet de référence (route $v s$ arbre), mais aussi de son type (route $v s$ chemin) et l'inconstance des locuteurs, liée à une expression «naïve » des localisations (Egenhofer et Mark, 1995). Le choix d'un seuil trop restrictif ou trop lâche sera nécessairement source d'un grand nombre de faux, positifs ou négatifs.

Pour pallier ce problème, la solution la plus répandue dans la littérature consiste à construire des objets spatiaux vagues, i.e. des objets aux limites non nettes, capables de prendre en compte l'imprécision. Ce type d'objet, présenté ci-dessous, permet de délimiter des zones de façon non bivalente.

\section{Modélisation des objets spatiaux vagues}

Cette partie présentera un aperçu des différents modèles et implémentations proposés dans la littérature pour modéliser des objets spatiaux vagues (Dilo, 2006). Nous en détaillerons ensuite une sélection, au regard de notre problématique.

\section{1 État de l'art}

Le recours à la modélisation d'objets vagues naît de la nécessité de modéliser des objets spatiaux dont les caractéristiques rendent caduque la modélisation à l'aide d'objets spatiaux aux frontières nettes. C'est notamment le cas lorsque l'on cherche à modéliser des objets dont la frontière ne peut pas être définie de manière précise. Citons l'exemple de la rivière polluée proposé par Dilo (2006). Dans ce cas, il est difficile de définir la zone de la rivière polluée puisque le degré de pollution décroit 
Objets et relations spatiales composites pour interpréter un référencement spatial indirect. 7

graduellement à partir de la source. Fixer la frontière d'un tel objet conduira à un choix discutable, puisque dépendant d'un seuil fixé empiriquement.

De nombreuses approches et implémentations des objets spatiaux vagues ont été proposées dans la littérature. Clementini (2008) distingue trois catégories : les modèles définissant des objets aux frontières étendues ( objects with broad boundaries»), les modèles basés sur la théorie des ensembles flous (Zadeh, 1965) et ceux basés sur la théorie des probabilités.

La catégorie des modèles aux frontières étendues regroupe les modélisations d'objets spatiaux remplaçant les traditionnelles frontières nettes par des frontières non nettes. Le modèle egg-yölk proposé par Cohn et Gotts (1996) est probablement le plus connu dans cette catégorie. On peut également citer le modèle min-max (Bejahoui et al, 2009) qui est une amélioration du modèle egg-yölk ou le modèle de Clementini (2008). Selon la définition proposée par Clementini (2008), un objet aux frontières vagues est composé de deux régions $r a$ et $r b$ avec $r a \subseteq r b$. $r r a$ correspond à la frontière intérieure et $\partial r b$ à la frontière extérieure de l'objet spatial. La frontière de l'objet spatial est alors définie comme la zone comprise entre la frontière intérieure et la frontière extérieure.

Les modèles basés sur la théorie des ensembles flous (voir la Figure 1), tel que celui proposé par Schneider (1999), proposent de faire une analogie entre un objet spatial et un ensemble flou. Zadeh (1969) définit un ensemble flou $A$ comme un couple composé d'un ensemble $X$ et d'une fonction $f_{A}$ nommée fonction d'appartenance. La fonction $f_{A}$, associe à chaque élément de $\mathrm{X}$ une valeur comprise dans l'intervalle $[0,1]$, nommée degré d'appartenance :

$$
A=\left(X, f_{A}\right), \operatorname{avec} f_{A}: X \rightarrow[0,1]
$$

L'ensemble des éléments ayant un degré d'appartenance non nul permet de définir le support $S$ de l'ensemble (cf. eq. 6). Le noyau $N$ de l'ensemble correspond, quant à lui, à l'ensemble des éléments ayant un degré d'appartenance égal à 1 . Le noyau, N, et le support, $\mathrm{S}$, de l'ensemble flou $A$ sont des ensembles nets.

$$
\begin{aligned}
& N(A)=\left\{x \in X \mid f_{A}(x)=1\right\} \\
& S(A)=\left\{x \in X \mid f_{A}(x)>0\right\}
\end{aligned}
$$

Le noyau et le support de l'ensemble A sont des cas particuliers de coupes-alpha (alpha-cuts) définies comme :

$$
A^{\alpha}=\left\{x \in X \mid f_{A}(x)>\alpha\right\}
$$

Schneider (1999) définit un équivalent flou à tous les objets spatiaux habituels. Ainsi, les points, les lignes et les polygones sont respectivement déclinés en points, lignes et polygones flous. Un point flou $p f$ est défini comme un point ayant un degré d'appartenance non nul. Par extension une ligne et une région floue lf et $r f$ sont définies comme des ensembles particuliers de points flous avec des contraintes spécifiques. Dilo et al. (2007) ont proposé une implémentation de ce modèle. Un 
point flou est implémenté comme un triplet contenant les coordonnées du point et son degré d'appartenance. De manière analogue au modèle théorique, une ligne floue est une liste ordonnée de points flous. Les auteurs proposent de reconstruire une approximation de la ligne floue à l'aide d'une interpolation linéaire entre chaque couple de points. La même démarche est proposée pour l'implémentation des régions floues. Ainsi, les limites du noyau et du support de la région floue sont stockées sous la forme de polylignes nettes et pour chaque point de la région floue une approximation du degré d'appartenance est calculée par interpolation.

Vanegas et al. (2011) proposent également de modéliser des objets spatiaux flous, mais avec une implémentation raster. L'espace traité est divisé en cellules de même taille pour lesquelles est calculé un degré d'appartenance. Cette approche permet de construire en extension l'ensemble des positions validant les règles de construction définies. Cette approche présente l'avantage de faciliter les opérations inter-objets, mais sa précision est fortement dépendante de la résolution choisie.

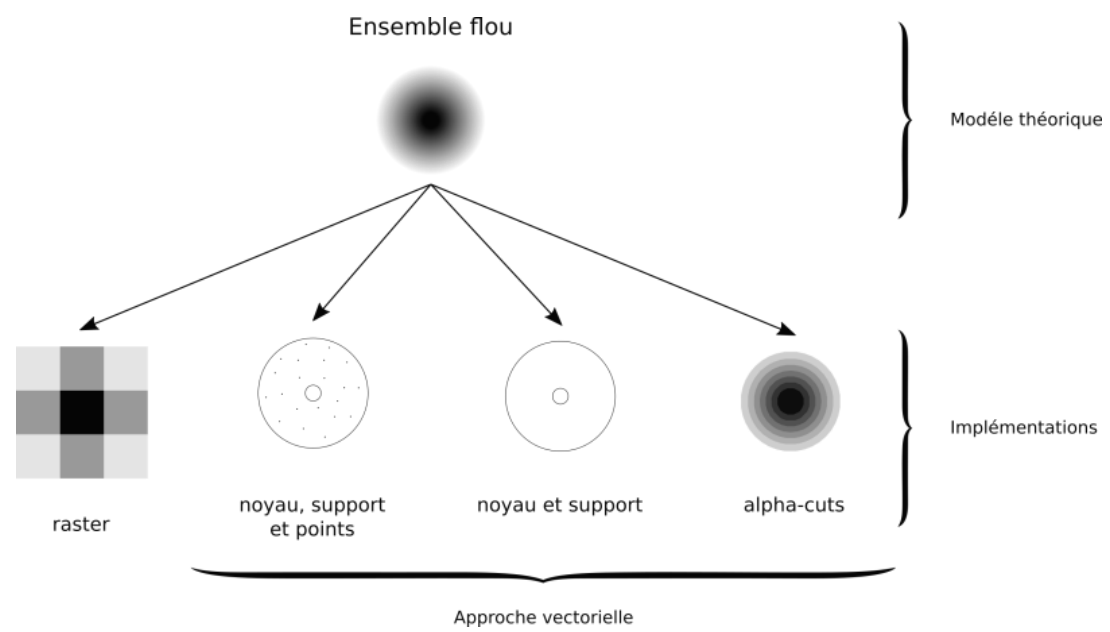

Figure 1. Implémentations d'objets spatiaux flous

Une autre proposition, basée sur la théorie des ensembles flous, est d'enregistrer une approximation des objets spatiaux flous à l'aide de coupes-alpha (de Runz, 2008 ; Zoghlami et al., 2016). Cette implémentation offre la possibilité de stocker des objets spatiaux flous sans recourir à des développements spécifiques, contrairement à l'approche de Dilo et al. (2007), mais les opérations inter-ensembles (union, intersection, etc.), demandent toutefois des développements ad hoc, contrairement à l'approche raster, en contrepartie plus volumineuse.

Enfin, Tøssebro et Nygård (2002) proposent une troisième catégorie de modèles, basée sur la théorie des probabilités. De manière analogue au travail de Schneider (1999), ils proposent d'étendre les concepts de points, lignes et régions en leurs équivalents incertains. Dans ce cas, les points, lignes et régions sont inclus au sein d'une région nette, définissant l'ensemble des positions possibles. L'incertitude est modélisée à l'aide d'une fonction de masse, attribuant à chaque point de la région nette une probabilité d'accueillir l'objet spatial incertain. 
Objets et relations spatiales composites pour interpréter un référencement spatial indirect. 9

\subsection{Comparaison et choix d'un modèle et de son implémentation}

Le choix de notre approche est contraint par le besoin d'agréger l'information issue de la modélisation de plusieurs éléments de localisation, mais aussi de plusieurs relations spatiales atomiques. Tous les modèles présentés permettent ce type de manipulation, mais tous n'offrent pas la même simplicité.

Les modèles discrets, tels que min-max ou egg-yölk, ont pour principal défaut leur première qualité, à savoir la simplicité. Ils ne permettent pas de prendre en compte la variation d'appartenance entre leurs deux frontières. Le calcul du degré d'appartenance d'une position à une région floue n'est donc envisageable qu'au prix d'une hypothèse sur la variation du degré d'appartenance entre les frontières. Les modèles proposés par Schneider (1999), Tøssebro et Nygård (2002) permettent, quant à eux, de modéliser une variation quelconque du degré d'appartenance. Bien entendu, les implémentations de ces modèles échantillonnent cette valeur, mais la précision de cette dernière, notamment dans les cas de variation non linéaire du degré d'appartenance, reste plus importante que celle des modèles min-max ou eggyölk.

Parmi les modèles permettant de modéliser des variations non linéaires, nous avons choisi de travailler avec un modèle fondé sur la théorie des ensembles flous, ces modèles étant les plus adaptés à nos contraintes. Toutefois, il est nécessaire de choisir une implémentation parmi celles présentées précédemment. Nous avons décidé de ne pas travailler à partir du modèle présenté par Dilo (2007), bien qu'il s'agisse d'une approche intéressante, puisque combinant à la fois les qualités d'une approche vectorielle (définition des frontières) et matricielle (meilleure précision de l'échantillonnage du degré d'appartenance). Cependant, cette implémentation est peu efficace pour manipuler des opérations inter-ensembles, fortement utilisées dans notre cas. Pour trancher entre une implémentation sous la forme d'alpha-cuts (de Runz, 2008), solution la plus économe en mémoire et une implémentation raster (Vanegas, 2011), plus précise, nous avons choisi de confronter ces deux approches sur un cas particulier, celui de l'élément de localisation : "je suis sous une ligne à haute tension ».

\section{Cas d'application}

En vue d'une sélection nous allons présenter une comparaison de deux implémentations des objets spatiaux flous présentés ci-dessus, à savoir les alpha-cuts (de Runz, 2008) et l'approche raster (Vanegas, 2011). Ces deux implémentations seront appliquées au même cas, issu d'une situation réelle. La victime a indiqué qu'elle était située "sous une ligne à haute-tension», élément de localisation correspondant au triplet (je, (suis, sous), une ligne à haute tension). 


\subsection{Décomposition de la relation spatiale}

Comme illustré dans la section 3.1, être sous un objet, quelle que soit sa nature, implique que le sujet soit proche et situé à une altitude inférieure à l'objet auquel il se réfère. Dans notre contexte, caractérisé par des dénivelés pouvant être importants, ces deux contraintes sont fortement indépendantes. Nous définissons donc deux règles : 1) le sujet doit être proche de l'objet de référence et 2) le sujet doit avoir une altitude inférieure à l'objet de référence (cf. Figure 2).

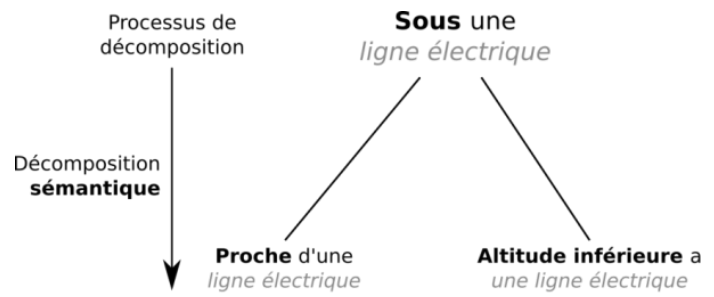

Figure 2 Décomposition du triplet (je, (suis, sous), une ligne électrique)

Il est nécessaire de fixer des seuils pour transformer ces descriptions qualitatives en règles exploitables. Ici, nous nous contenterons de fixer des seuils génériques, laissant le traitement de leurs variabilités locales à une exploration ultérieure. Le recours aux objets spatiaux flous permet de prendre en compte l'imprécision inhérente à ce type de transformations. Il est toutefois nécessaire de fixer manuellement deux seuils : $d_{S}$ représentant la distance à partir de laquelle une position n'est plus proche d'une ligne à haute-tension (limite du support) et $d_{N}$, la distance en deçà de laquelle une position est proche d'une ligne à haute tension (limite du noyau). La Figure 3 présente ces seuils et la forme de la fonction d'appartenance qui en découle.

L'ensemble des positions proches d'une ligne à haute tension est défini par la fonction d'appartenance suivante :

$$
f_{1}(d)=\left\{\begin{array}{c}
1 \text { si } d<d_{N} \\
0 \text { si } d>d_{S} \\
1-\frac{d-d_{N}}{d_{S}-d_{N}} \text { sinon }
\end{array}\right.
$$

Où $d$ représente la distance à l'axe médian de la ligne à haute tension. Pour cette application, en collaboration avec le PGHM de Grenoble, les seuils retenus sont de $50 \mathrm{~m}$ pour $d_{N}$, et de $100 \mathrm{~m}$ pour $d_{S}$.

De la même manière, l'ensemble contenant toutes les positions situées à une altitude inférieure à celle d'une ligne à haute tension est défini à travers la fonction d'appartenance suivante :

$$
f_{2}(H, h)=\left\{\begin{array}{l}
1 \text { siH }-h>h_{N} \\
0 \text { siH }-h<h_{S} \\
\frac{(H-h)-h_{N}}{h_{S}-h_{N}} \text { sinon }
\end{array}\right.
$$


Avec $H$ la hauteur de la ligne à haute-tension la plus proche et $h$ l'altitude de la position, $h_{N}$ et $h_{S}$ la différence entre $H$ et la hauteur, respectivement, du noyau et du support. Les seuils ont été fixés empiriquement à $5 \mathrm{~m}$ pour $h_{N}$ et à $0 \mathrm{~m}$ pour $h_{S}$ (soit le sommet du pylône).

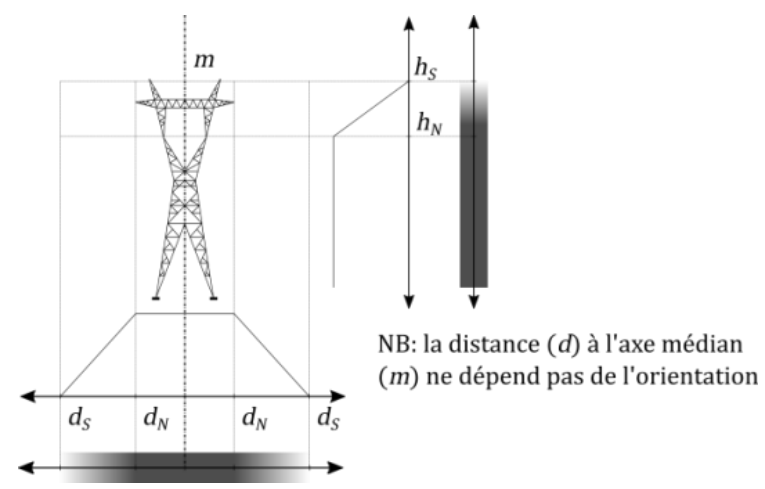

Figure 3. Définition de l'ensemble flou « je suis sous une ligne électrique »

Enfin, l'ensemble contenant toutes les positions situées sous une ligne à haute tension est défini à partir des fonctions d'appartenances décrites par les équations (8) et (9) de la manière suivante :

$$
f_{3}(d, H, h)=\min \left(f_{1}(d), f_{2}(H, h)\right)
$$

Où min représente l'opérateur d'intersection proposé par Zadeh (1965), défini comme le minimum des deux valeurs d'appartenance.

\subsection{Application}

Les deux implémentations présentées, portent sur la région de Bourg d'Oisans, au sud-est de Grenoble. Deux sources de données ont été utilisées. Les lignes à haute tension, ainsi que leurs pylônes proviennent de la BD TOPO produite par l'IGN. Le MNT, d'une résolution de cinq mètres, est quant à lui extrait de la BD ALTI de 2017.

La première implémentation testée repose sur la méthodologie proposée par Vanegas et al. (2011). L'ensemble des positions étudiées, délimité par une aire d'étude précédemment définie, est échantillonné par une grille à la résolution calquée sur le MNT. Nous calculons alors la valeur du degré d'appartenance pour chacune de ces cellules, permettant ainsi de définir un ensemble flou en extension. Dans notre cas, nous construisons trois rasters (Figure 4), représentant autant d'ensembles flous, comme défini dans les équations 8, 9 et 10 . Nous avons choisi de rastériser la couche ligne électrique de la BD TOPO, contenant l'ensemble des portions aériennes de lignes à haute et très haute tension. À l'aide de cette couche intermédiaire nous mesurons la distance planimétrique séparant chaque cellule du raster de la cellule la plus proche occupée par une ligne électrique. Cette mesure de 
distance est utilisée comme paramètre de la fonction d'appartenance présentée en Eq. 8 et dont le résultat est présenté par la Figure 4(a).

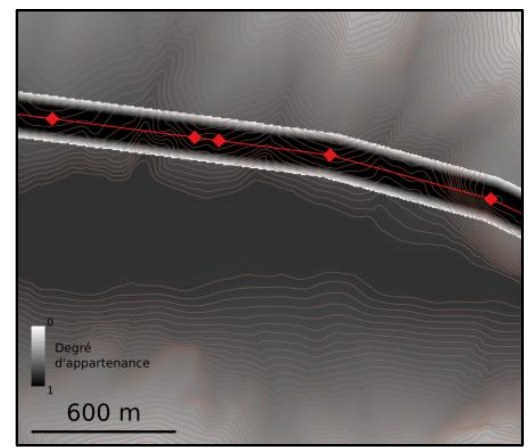

(a) Degré d'appartenance à l'ensemble des positions proches d'une ligne à haute tension

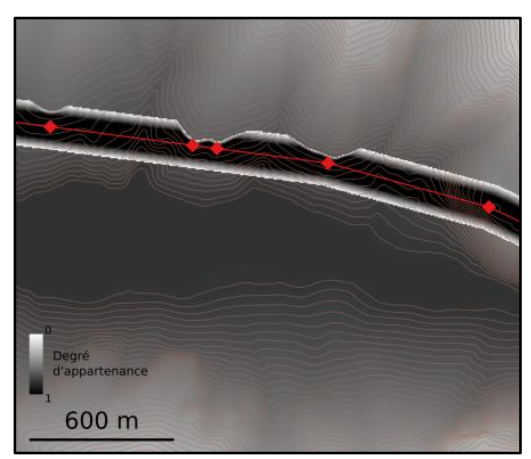

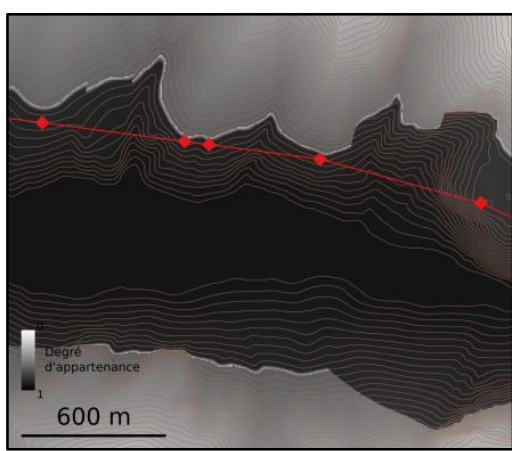

(b) Degré d'appartenance à l'ensemble des positions situées à plus basse altitude qu'une ligne à haute tension

\section{(c) Degré d'appartenance à} l'intersection des ensembles

Figure 4. Modélisation d'objets spatiaux flous : approche raster

Le calcul du degré d'appartenance à l'ensemble des positions situées à une altitude inférieure à celle d'une ligne à haute-tension consiste à calculer la différence entre l'altitude de la position et celle des lignes à haute tension. Il s'est avéré nécessaire de calculer une estimation de la hauteur de la ligne à haute tension en toute cellule traversée par une ligne à haute tension. Nous avons estimé cette valeur à l'aide d'une interpolation linéaire limitée à deux points (les deux pylônes les plus proches de la cellule), avant de rogner ce résultat à l'aide de l'empreinte au sol des lignes à haute tension précédemment calculées. Cette nouvelle donnée, enrichissant la rastérisation des lignes à haute-tension a été utilisée comme paramètre de la fonction d'appartenance présentée en Eq. 9. La Figure 4(b) représente le degré d'appartenance ainsi calculé.

Les deux ensembles flous (Figures. 4(a) et 4(b)) permettent de construire l'ensemble représentant les positions situées sous une ligne électrique. La fonction d'appartenance de ce dernier prend trois paramètres (cf. Eq. 10). Nous remarquons que la zone construite est sensiblement différente de celle que nous aurions obtenue si nous n'avions pris en compte la seule distance (Figure 4(a). Certaines positions, 
très proches d'une ligne à haute-tension peuvent, en effet, être situées à des altitudes dépassant fortement le sommet des pylônes avoisinants, démontrant l'intérêt de prendre en compte la différence d'altitude.

La seconde implémentation testée est basée sur l'approche utilisée par de Runz (2008) et Zoghlami et al. (2016) nécessitant une approche vectorielle. Cependant, aucun système d'information géographique ou plus généralement logiciel permettant la manipulation de données spatiales vectorielles ne permet, du moins à notre connaissance, de manipuler des géométries tridimensionnelles telles que celles que nous souhaitons construire. Nous nous sommes donc orientés vers la bibliothèque CGAL (CGAL Project, 2019), spécialisée dans la géométrie algorithmique.

La démarche suivie a consisté à créer des polyèdres, représentant les alpha-cuts tridimensionnelles de l'ensemble flou contenant les positions sous une ligne électrique. Comme illustré par la Figure 5, pour chaque segment $\mathrm{S}$ d'une ligne à haute tension, nous définissons six plans, délimitant autant de demi-espaces et dont l'intersection permet de définir un volume fini. Le premier plan $(\mathrm{P} 1)$ est parallèle au plan $N$ incluant le segment $S$ et une droite horizontale perpendiculaire à $S$. La différence d'altitude $(\Delta \mathrm{h})$ entre les plans P1 et $N$ varie en fonction de l'alpha-cut modélisée. Le second plan $(\mathrm{P} 2)$ représente la limite inférieure du polyèdre, son $\mathrm{z}$ est nul et constant. Les plans $\mathrm{P} 3$ et $\mathrm{P} 4$ représentent l'avant et l'arrière du polyèdre (par rapport à la direction de la ligne électrique). Ils sont perpendiculaires au plan P2 et passent respectivement par le premier et dernier point du segment $\mathrm{S}$. Enfin, les plans P5 et P6 représentent la gauche et la droite du polyèdre. Ils sont verticaux et parallèles au segment $\mathrm{S}$. Comme pour le plan P1, leur distance $(d)$ au segment $S$ varie en fonction de l'isovolume modélisé.

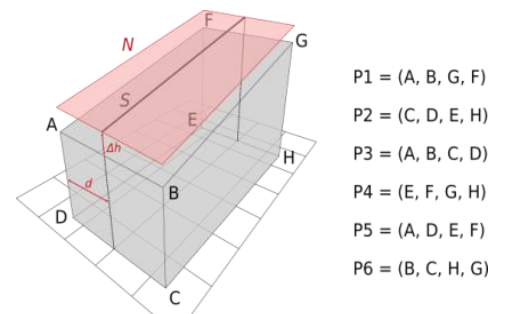

Figure 5. Plans utilisés pour définir une alpha-cut

Pour modéliser les alpha-cuts, nous faisons varier la hauteur du plan P2 et l'éloignement des plans P5 et P6 par rapport au segment S. En identifiant les valeurs de hauteur et d'éloignement correspondant au degré d'appartenance défini, on peut construire les alpha-cuts désirées. La Figure 6(a) montre trois alpha-cuts $(0 ; 0,5$ et 1) construites à partir d'un segment de ligne à haute-tension. En intersectant ces géométries avec le MNT (Figure 6(b)), nous pouvons projeter les alpha-cuts au sol et ainsi construire une carte en deux dimensions (Figure 6(c)) représentant les alphacuts de l'ensemble flou des positions situées sous une ligne haute-tension dans notre aire d'étude. 
(a) alpha-cuts pour un segment de ligne à haute tension
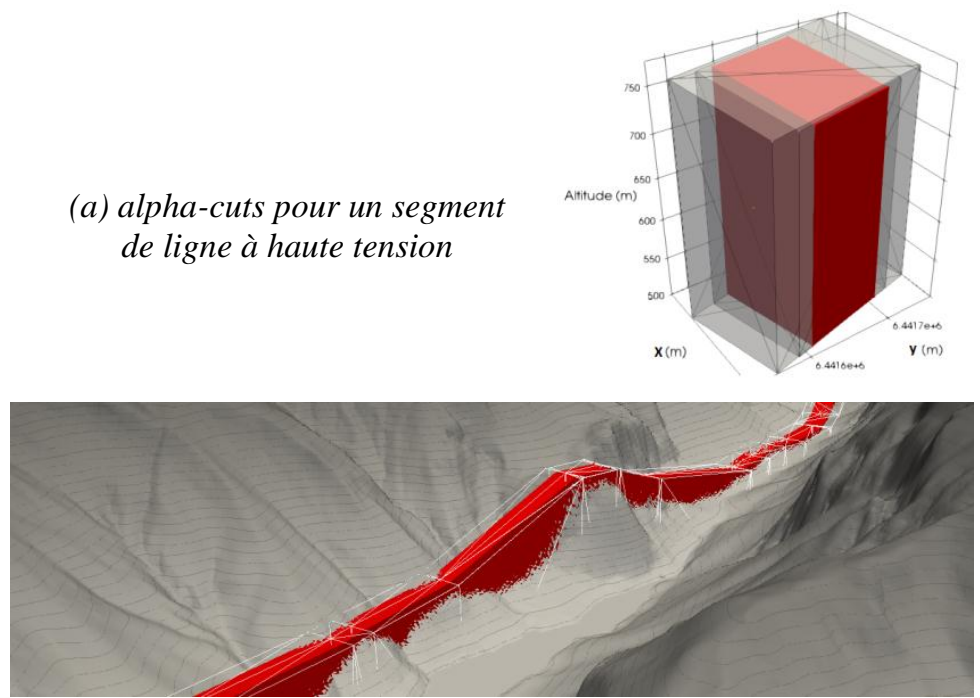

(b) Intersection des alpha-cuts avec le MNT

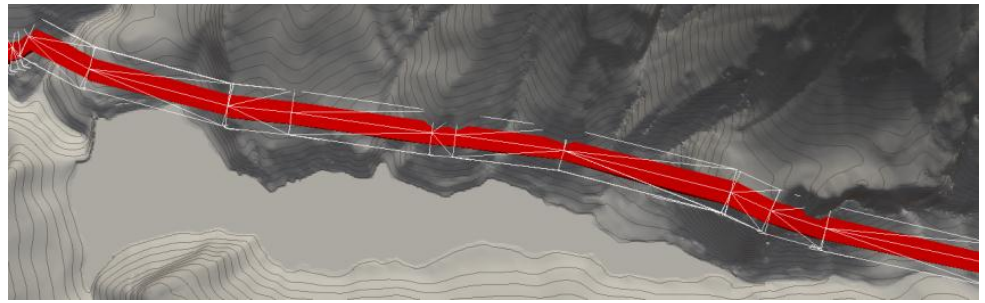

(c) Vue zénithale des alpha-cuts

Figure 6. Modélisation d'objets spatiaux flous : approche vectorielle

\subsection{Comparaison}

Cette double implémentation d'un même élément de localisation a fait apparaitre les avantages et limites des deux approches. Notre besoin de travailler en trois dimensions est source de nombreuses complications liées à la difficulté de manipulation de géométries 3D. L'implémentation en alpha-cuts a nécessité l'utilisation de la bibliothèque CGAL (aucun SIG n'étant envisageable) et un travail de développement non négligeable (cf. tableau 1), pour un résultat comparable à celui de l'approche raster (cf. figure 7).

La figure 7 illustre les différences d'estimation de la zone «sous une ligne électrique » entre l'approche raster et l'approche par alpha-cuts. Ici, les différences, représentées sous la forme d'un raster, dont la maille correspond à celle du MNT, sont de l'ordre du pixel. Les deux méthodes aboutissent à des résultats similaires. On peut cependant remarquer que l'approche par raster tend, comparativement à l'approche par alpha-cuts, à surévaluer la zone correspondant à l'élément de 
localisation modélisé. Ainsi, 5,4\% de la surface du noyau et 9,4\% de la surface du support estimées par l'approche raster sont absentes de l'estimation par alpha-cuts, contre $2,1 \%$ de la surface du noyau et $3,6 \%$ de la surface du support dans le cas contraire. Mais cette comparaison illustre avant tout les artéfacts nés d'une implémentation imparfaite. L'approche raster offre de meilleurs résultats à proximité de la jointure des segments de ligne, notre implémentation de l'approche par alphacuts n'opérant pas de traitement particulier en vue de modéliser ces jointures. Inversement, l'approche par alpha-cuts est plus efficace lorsque l'estimation de la hauteur de la ligne à haute tension utilisée par l'approche raster est trop grossière, ce qui est notamment le cas lorsque plusieurs lignes sont à proximité. Il s'agit toutefois de problèmes d'implémentation pouvant être corrigés dans les deux cas, même si la quantité de travail supposée nécessaire est plus importante dans le cas de l'approche par alpha-cuts.
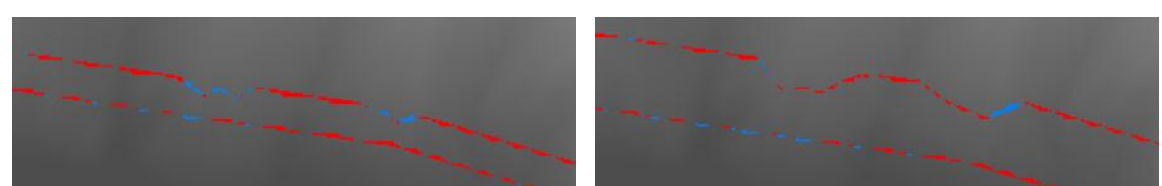

Figure 7 Comparaison des zones construites à l'aide des approches raster et alpha-cuts. En rouge figurent les pixels uniquement présents dans l'estimation par raster et en bleu les zones uniquement présentes dans l'estimation par alphacuts. L'image de gauche représente la comparaison des deux noyaux et celle de droite la comparaison des deux supports

Dans le cas où les deux approches ne produisent pas d'artefacts (e.g. Figure 7), les deux estimations sont extrêmement proches, ce qui nous pousse naturellement à privilégier l'approche présentant le meilleur ratio qualité de l'estimation / difficulté de mise en place. Il nous semble donc plus pertinent de travailler, comme le propose Vanegas et al. (2011), à l'aide de rasters. Les règles utilisées pour définir l'ensemble flou à l'aide de l'approche raster présentent l'avantage d'être simples et peu nombreuses, ce qui laisse également supposer une meilleure capacité de généralisation de la méthodologie.

Tableau 1 Comparaison des temps de développement, d'exécution et estimation des temps d'amélioration des deux approches présentées.

\begin{tabular}{|c|c|c|}
\hline & Raster & Alpha-cuts \\
\hline Temps de développement & $5 \mathrm{~h}$ & $60 \mathrm{~h}$ \\
\hline Temps d'exécution & $2 \mathrm{~min}$ & $5 \mathrm{~min}$ \\
\hline $\begin{array}{c}\text { Estimation de l'importance des } \\
\text { développements à venir }\end{array}$ & + & +++ \\
\hline
\end{tabular}




\section{Extension à d'autres objets de référence}

Comme nous l'expliquons dans la section 3, la nature de l'objet de référence considéré influe sur la sémantique de la relation spatiale qui lui est associée dans un élément de localisation. C'est par exemple le cas de la relation spatiale «sous » dont la sémantique est différente pour les objets de référence "ligne à haute-tension » et "route ». Lorsque l'objet de référence est composite, une sémantique différente de la même relation spatiale peut s'appliquer aux différentes composantes de l'objet de référence. La modélisation d'un tel élément de localisation nécessite alors deux phases de décomposition, une première méréologique et une seconde sémantique. Pour illustrer cette démarche, nous proposons de modéliser l'élément de localisation "je suis sous une télécabine », qui, comme l'élément de localisation "je suis sous une ligne électrique » précédemment traité, est basé sur un cas réel.

\subsection{Décomposition de l'objet de référence « télécabine »}

Les points de départ et d'arrivée d'une télécabine, comme ceux de toute remontée mécanique téléportée, prennent la forme de deux gares, qui, de par leur taille et la rareté des bâtiments à haute altitude, sont plus saillantes que la ligne d'une remontée mécanique. Un requérant peut donc décrire sa position à partir du point d'arrivée d'une télécabine, c'est-à-dire d'un bâtiment, et l'apparenter, par métonymie, à la télécabine.

Nous proposons donc de décomposer l'objet de référence "télécabine » en deux éléments, d'une part les gares et d'autre part la ligne (cf. Figure 8). L'objet de référence "ligne de télécabine » pourrait être décomposé à son tour, par exemple en pylônes et câble, mais un tel niveau de détail est ici excessif. Cette décomposition nous conduit donc à modéliser deux éléments de localisation différents, "je suis sous une ligne de télécabine » et « je suis sous une gare de télécabine ».

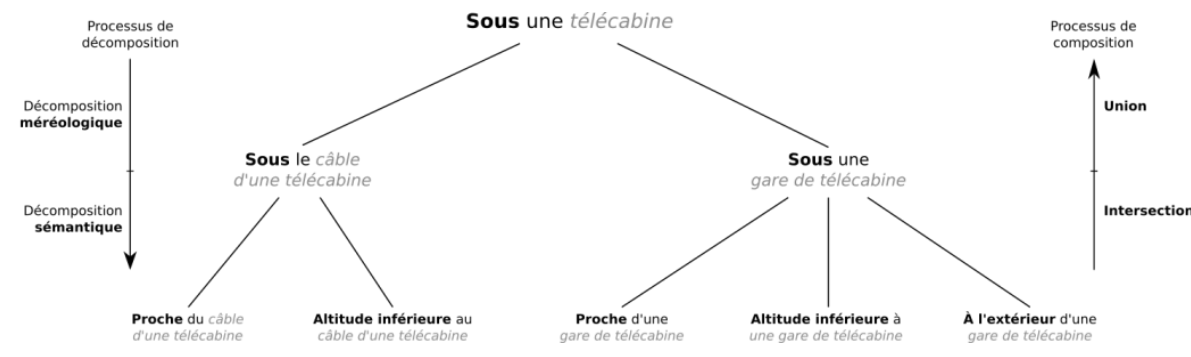

Figure 8 Décomposition de l'élément de localisation «je suis sous une télécabine »

\subsection{Spatialisation d'un élément de localisation comprenant un objet de référence composite}

Si l'on peut supposer que la sémantique de la relation spatiale «sous» est identique pour les objets de référence «ligne électrique » et «ligne de télécabine », 
il n'en va pas nécessairement de même pour l'objet de référence "gare de télécabine». La spatialisation de l'élément de localisation "je suis sous une télécabine » nécessite donc de prendre en compte cette variation sémantique. Nous allons maintenant étudier séparément « sous une ligne de télécabine » et "sous une gare de télécabine ».

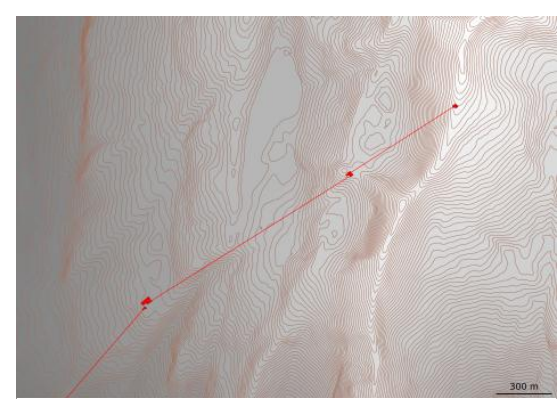

(a) Objet de référence utilisé : une ligne de télécabine

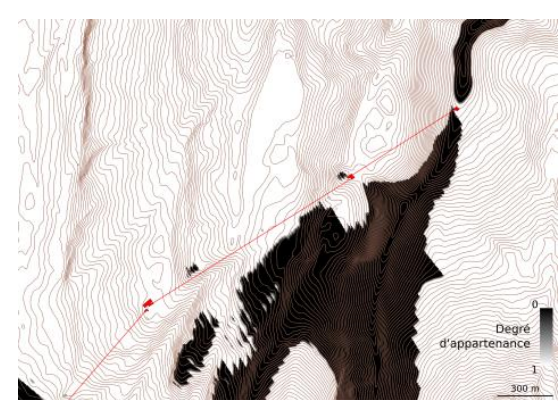

(c) Degré d'appartenance à

l'ensemble des positions situées à plus basse altitude que cette ligne de télécabine

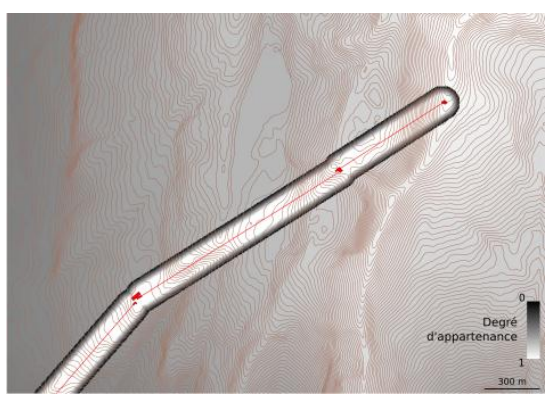

(b) Degré d'appartenance à l'ensemble des positions proches de cette ligne de télécabine

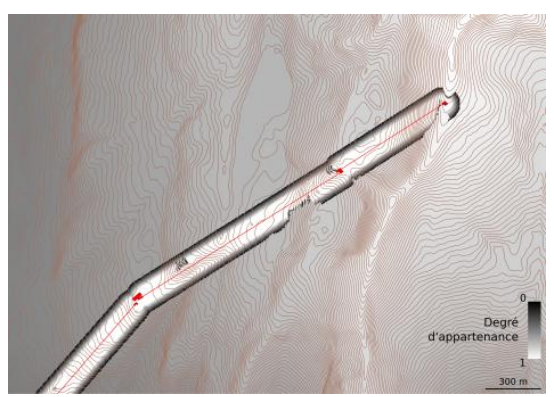

(d) Degré d'appartenance à

l'intersection des ensembles

Figure 9 Spatialisation de l'élément de localisation «je suis sous une ligne de télécabine »

La spatialisation de l'élément de localisation «je suis sous la ligne d'une télécabine » peut être effectuée à l'aide de la méthode précédemment proposée pour l'élément de localisation "je suis sous une ligne électrique », la même sémantique de la relation spatiale "sous »s'appliquant aux deux objets de référence. N'ayant pas plus d'éléments que précédemment pour fixer les seuils des fonctions d'appartenance, nous avons utilisé les mêmes valeurs que précédemment, soit, pour la proximité : $50 \mathrm{~m}$ comme limite du noyau et $100 \mathrm{~m}$ comme limite du support et pour la différence d'altitude : $5 \mathrm{~m}$ en-dessous du câble pour la limite du noyau et la hauteur du câble pour la limite du support. La modélisation résultante, testée sur une 
ligne de télécabine réelle (composée de trois tronçons et extraite de la BDTopo), est visible sur la Figure 9.

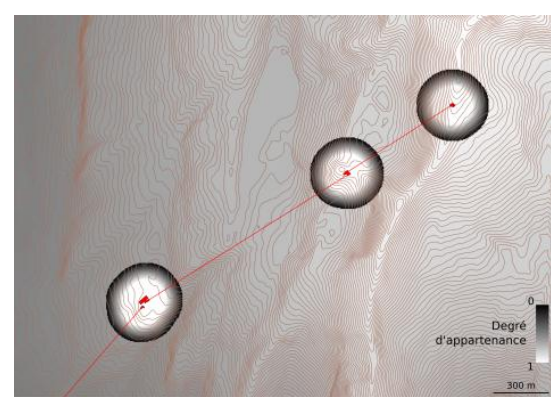

(a) Degré d'appartenance à l'ensemble des positions proches d'une gare de télécabine

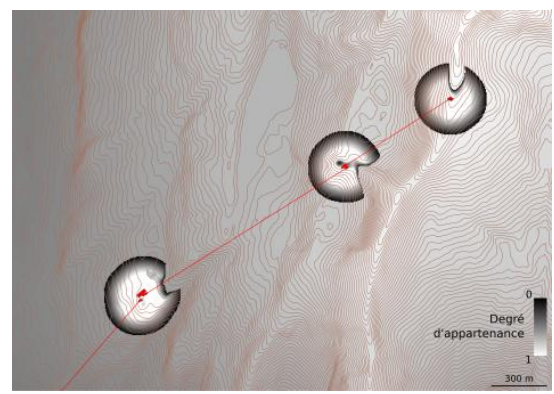

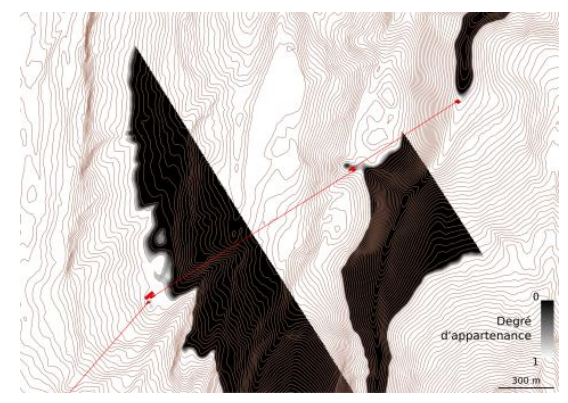

(b) Degré d'appartenance à l'ensemble des positions situées à une altitude inférieure à celle d'une gare de télécabine

\section{(c) Degré d'appartenance à}

l'intersection des ensembles

Figure 10 Spatialisation de l'élément de localisation « je suis sous une gare de télécabine »

L'élément de localisation "je suis sous une gare de télécabine » a, quant à lui, une sémantique différente. Contrairement à une ligne électrique, on ne peut pas être situé à la parfaite verticale d'un bâtiment, mais nous n'avons pas pris en compte cet aspect dans nos modélisations. Cependant, nous supposons que les règles de proximité et de différence d'altitude utilisées jusqu'ici restent pertinentes, autrement dit, que l'on soit sous un câble ou sous un bâtiment, il est nécessaire que l'objet de référence soit proche et plus haut que le sujet.

Bien que l'élément de localisation "je suis sous une gare de télécabine » soit modélisé avec les mêmes relations spatiales atomiques que les éléments de localisation «je suis sous une ligne électrique» et "je suis sous un câble de télécabine", il nous a semblé nécessaire de modifier les seuils des fonctions d'appartenance pour prendre en compte la plus grande saillance des bâtiments. Une fois encore, le choix de ces seuils est empirique. Pour le critère de proximité, nous avons choisi de le fixer à $100 \mathrm{~m}$ pour la limite du noyau et $200 \mathrm{~m}$ pour la limite du support. Quant à la différence d'altitude, le seuil du noyau a été fixé à $5 \mathrm{~m}$ en dessous du bâtiment et celui du support à $5 \mathrm{~m}$ au-dessus (Figure 10). 
Une fois que les éléments de localisation «je suis sous une gare de télécabine »et «je suis sous une ligne de télécabine» ont été modélisés indépendamment, on peut modéliser l'élément de localisation "je suis sous une télécabine » en unissant les deux ensembles (cf. Figure 11).

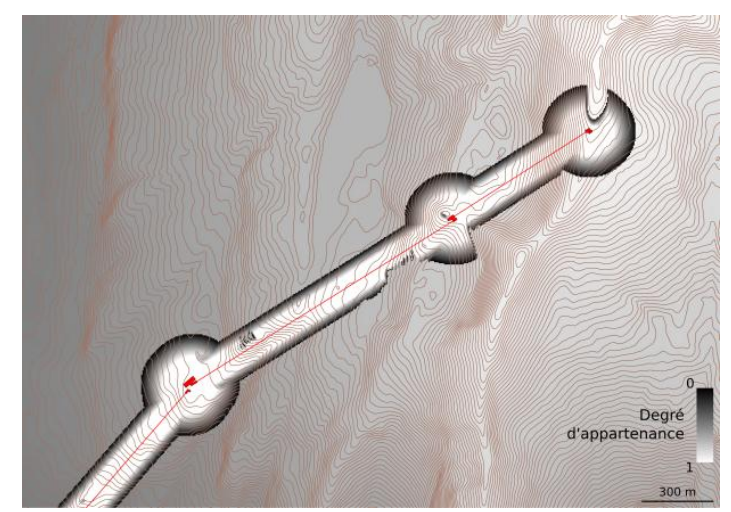

Figure 11 Degré d'appartenance à l'union des ensembles (fig. 9d et 10c)

\section{Analyse de sensibilité}

Comme nous l'avons mentionné dans les sections 5 et 6 , la définition des seuils a été réalisée à partir d'un paramétrage empirique des fonctions d'appartenance. Afin d'analyser l'influence des seuils sur l'ensemble des positions construit lors de la spatialisation d'un élément de localisation, nous avons effectué une analyse de sensibilité des seuils des fonctions d'appartenance utilisées pour spatialiser l'élément de localisation «je suis sous une télécabine » décrit dans la section 6.

Pour faciliter la comparaison entre les différentes exécutions du modèle, nous avons travaillé sur une zone de $10 \mathrm{~km}^{2}$, centrée sur une ligne de télécabine (déjà visible sur les figures 10 et 11). La démarche suivie est basée sur la méthode dite «de Morris » (Morris, 1991). Cette dernière permet d'identifier les paramètres influant sur une sortie à l'aide d'un plan d'expérience faisant varier les paramètres $p$ de la simulation un par un, dans le but d'offrir une bonne couverture de l'espace des paramètres. Cette méthode nécessite de sélectionner un nombre $t$ d'échantillons et renvoie un plan d'expérience de $t^{*}(p+1)$ simulations. La mesure de l'influence de la variation d'un paramètre sur la sortie nécessite la définition d'une métrique adaptée à l'évaluation de la problématique. La comparaison du plan d'expérience et de la métrique permet le calcul de deux indices, $\mu^{*}$ qui mesure la sensibilité du paramètre, (une forte valeur indique une forte influence du paramètre sur les résultats du modèle) et $\sigma$ qui mesure les interactions entre les paramètres (sa valeur augmente en présence d'interactions entre paramètres ou des effets non linéaires). La valeur de ces indices est à considérer relativement aux autres valeurs et non dans l'absolu, puisque dépendante du domaine de la métrique sélectionnée. 
Pour cette analyse, nous avons identifié 8 paramètres (cf. tableau 2) et fixé le nombre de trajectoires à 1000 (soit 9000 simulations), valeur présentant un bon compromis entre couverture de l'espace des paramètres et temps de calcul.

Tableau 2 Variation des paramètres utilisés pour l'analyse de sensibilité

\begin{tabular}{|c|c|c|}
\hline Paramètre & Description & Valeurs \\
\hline ldist $\left(\mathrm{d}_{\mathrm{N}}\right)$ & Distance du noyau à la ligne & De 0 à 250 \\
\hline ldistR $\left(\mathrm{d}_{\mathrm{S}}\right)$ & $\begin{array}{l}\text { Distance de la limite du support au noyau pour la distance à } \\
\text { la ligne }\end{array}$ & De 1 à 1750 \\
\hline ldalt $\left(\mathrm{h}_{\mathrm{N}}\right)$ & Valeur du noyau pour la différence d'altitude à la ligne & De -25 à 25 \\
\hline $\operatorname{ldaltR}\left(\mathrm{h}_{\mathrm{S}}\right)$ & $\begin{array}{l}\text { Distance de la limite du support au noyau pour la différence } \\
\text { d'altitude à la ligne }\end{array}$ & De 1 à 50 \\
\hline bddist $\left(\mathrm{d}_{\mathrm{N}}\right)$ & Distance du noyau aux gares & De 0 à 250 \\
\hline $\operatorname{bdistR}\left(\mathrm{d}_{\mathrm{S}}\right)$ & $\begin{array}{l}\text { Distance de la limite du support au noyau pour la distance } \\
\text { aux gares }\end{array}$ & De 1 à 1750 \\
\hline bdalt $\left(\mathrm{h}_{\mathrm{N}}\right)$ & Valeur du noyau pour la différence d'altitude à la ligne & De -25 à 25 \\
\hline $\operatorname{bdaltR}\left(\mathrm{h}_{\mathrm{S}}\right)$ & $\begin{array}{l}\text { Distance de la limite du support au noyau pour la différence } \\
\text { d'altitude aux gares }\end{array}$ & De 1 à 50 \\
\hline
\end{tabular}

\subsection{Analyse de sensibilité basée sur la taille de la zone}

Une première métrique envisageable pour rendre compte de la variabilité du résultat (la zone floue obtenue) en fonction des paramètres en entrée est la part de l'aire totale occupée par l'ensemble des positions construit suite au processus de spatialisation (cf. Figure 11). Cette mesure ne permet pas de rendre directement compte de l'importance de la zone floue, puisque le support est considéré sans distinction de degré d'appartenance, mais elle permet néanmoins d'observer l'impact des paramètres ldist, bdist, ldist $R$ et, bdist $R$, (par le biais de l'indice $\mu^{*}$ ), sur la taille du support (cf. tableau 3). Les paramètres ldalt et bdalt ont également un impact sur cette métrique, mais les valeurs de l'indice $\mu^{*}$ (respectivement 0,030 et $0,010)$ indiquent que ce dernier est plus faible. Cette observation est confirmée par les résultats visibles sur les figures 9,10 et 11, où la forme de la zone construite par la spatialisation des deux éléments de localisation semble être principalement contrainte pas la règle de proximité et donc les paramètres ldist, ldalt, ldistR et ldaltR. Les valeurs importantes de l'indice $\sigma$ pour ces deux derniers paramètres sont dues au fait que la taille du support n'est pas directement un paramètre du modèle, mais est définie par la somme des variables ldist ou bdist avec, respectivement, les variables ldistR et bdistR. Dans l'ensemble, les paramètres en rapport avec la ligne de télécabine (ldist, ldistR et ldalt) ont un impact plus important que les paramètres en rapport avec les gares (bddist, bdistR et bdalt). Cet effet est dû à la différence 
d'emprise des deux types objets de référence. L'impact et l'interaction (indices $\mu^{*}$ et $\sigma)$ nuls des paramètres ldaltR et bdaltR sont dus à l'incapacité de la métrique choisie d'en percevoir une influence. L'augmentation de ces paramètres n'ayant, par construction, qu'une incidence limitée sur la taille du support. Cependant, ces paramètres peuvent avoir, à taille du support égale, une influence non négligeable sur la partie floue, mais seule l'utilisation d'une autre métrique peut répondre à ces questions.

Tableau 3 Résultats de l'analyse de sensibilité dont la métrique est : l'aire du support rapporté à l'aire totale

\begin{tabular}{|l|c|c|}
\hline \multicolumn{1}{|c|}{ Métrique utilisée } & \multicolumn{2}{|c|}{$\begin{array}{c}\text { Aire du support rapportée à l'aire totale de la zone } \\
\text { d'étude }\end{array}$} \\
\hline \multicolumn{1}{|c|}{ Paramètre } & $\mu^{*}$ & $\sigma$ \\
\hline ldist $\left(\mathrm{d}_{\mathrm{N}}\right)$ & 0,021 & 0,015 \\
\hline ldist $R\left(\mathrm{~d}_{\mathrm{S}}\right)$ & $\mathbf{0 , 1 3 8}$ & $\mathbf{0 , 0 8 0}$ \\
\hline ldalt $\left(\mathrm{h}_{\mathrm{N}}\right)$ & 0,030 & 0,020 \\
\hline ldalt $\left(\mathrm{h}_{\mathrm{S}}\right)$ & 0,000 & 0,000 \\
\hline bddist $\left(\mathrm{d}_{\mathrm{N}}\right)$ & 0,017 & 0,016 \\
\hline bdist $R\left(\mathrm{~d}_{\mathrm{S}}\right)$ & $\mathbf{0 , 1 1 1}$ & $\mathbf{0 , 0 8 3}$ \\
\hline bdalt $\left(\mathrm{h}_{\mathrm{N}}\right)$ & 0,010 & 0,010 \\
\hline bdalt $\left(\mathrm{h}_{\mathrm{S}}\right)$ & 0,000 & 0,000 \\
\hline
\end{tabular}

\subsection{Analyse de sensibilité basée sur l'indice de Shannon}

Pour compléter la première analyse de sensibilité nous avons réutilisé le même plan d'expérience avec une nouvelle métrique basée sur l'indice d'entropie de Shannon, initialement créé pour quantifier la redondance d'informations dans un signal.

L'indice de Shannon $\mathrm{H}(\mathrm{x})$ s'exprime de la manière suivante :

$$
H(x)=-\sum_{i}^{n} P i \log _{2} P i
$$

Où $P_{i}$ est la probabilité qu'une valeur appartienne à la catégorie $i$. Dans notre cas, on peut considérer des plages de degré d'appartenance comme des catégories, la probabilité $P_{i}$ d'appartenance à l'une de ces classes est égale au nombre de pixels appartenant à cette classe divisé par le nombre total de pixels. Cependant, cela nécessite de discrétiser le degré d'appartenance. Nous avons opté pour une discrétisation en dix classes, une première pour les degrés d'appartenance nuls, une seconde pour les degrés d'appartenance égaux à 1 (soit le noyau) et enfin 8 classes 
(de 0 à 1 exclus, avec un pas de 0,125) pour les valeurs floues. La valeur de l'indice de Shannon est nulle dans le cas où le degré d'appartenance de toutes les positions appartient à la même classe. Ce cas de figure peut se présenter lorsque la zone construite est vide, ou, au contraire, lorsque toutes les positions de l'aire d'étude appartiennent au noyau. L'indice de Shannon augmentera avec deux facteurs : l'aire du support et l'importance de la partie floue. À taille de support égale, l'indice de Shannon est plus important pour une zone dont le noyau est plus petit. Pour compléter cette analyse de sensibilité nous avons également calculé l'indice de Shannon sans prendre en compte les pixels dont le degré d'appartenance est nul. Dans ce cas, l'indice n'augmentera pas avec l'aire du support, mais uniquement avec l'importance de la zone floue.

Tableau 4 Résultats de l'analyse de sensibilité dont la métrique est : l'indice de Shannon

\begin{tabular}{|l|c|c|c|c|}
\hline \multicolumn{1}{|c|}{$\begin{array}{c}\text { Métrique } \\
\text { utilisée }\end{array}$} & \multicolumn{2}{|c|}{ Indice de Shannon } & $\begin{array}{c}\text { Indice de Shannon (sans degrés } \\
\text { d'appartenance nuls) }\end{array}$ \\
\hline Paramètre & $\mu^{*}$ & $\sigma$ & $\mu^{*}$ & $\sigma$ \\
\hline ldist $\left(\mathrm{d}_{\mathrm{N}}\right)$ & 0,149 & 0,257 & 0,131 & 0,078 \\
\hline ldist $R\left(\mathrm{~d}_{\mathrm{S}}\right)$ & $\mathbf{0 , 3 5 2}$ & $\mathbf{0 , 9 7 3}$ & $\mathbf{0 , 7 9 5}$ & $\mathbf{0 , 4 7 4}$ \\
\hline ldalt $\left(\mathrm{h}_{\mathrm{N}}\right)$ & 0,127 & 0,367 & 0,168 & 0,160 \\
\hline ldalt $\left(\mathrm{h}_{\mathrm{S}}\right)$ & 0,196 & 0,607 & 0,012 & 0,015 \\
\hline bddist $\left(\mathrm{d}_{\mathrm{N}}\right)$ & 0,107 & 0,258 & 0,099 & 0,088 \\
\hline bdist $R\left(\mathrm{~d}_{\mathrm{S}}\right)$ & $\mathbf{0 , 3 1 2}$ & $\mathbf{0 , 9 1 0}$ & $\mathbf{0 , 6 5 2}$ & $\mathbf{0 , 4 9 2}$ \\
\hline bdalt $\left(\mathrm{h}_{\mathrm{N}}\right)$ & 0,038 & 0,109 & 0,059 & 0,051 \\
\hline bdaltR $\left(\mathrm{h}_{\mathrm{S}}\right)$ & 0,048 & 0,196 & 0,004 & 0,004 \\
\hline
\end{tabular}

Les résultats de cette seconde analyse de sensibilité confirment que l'impact nul des paramètres ldaltR et bdaltR, était lié à la métrique choisie (cf. tableaux 3 et 4). Ces deux paramètres ont une influence sur la première et la seconde version de l'indice de Shannon, mais elle est très faible dans ce second cas $\left(\mu^{*}\right.$ de, respectivement, 0,012 et 0,004$)$, ce qui laisse supposer un impact sur la taille de la partie floue, mais au demeurant très faible. Une nouvelle fois, on peut remarquer que l'impact des paramètres jouant sur la ligne est un peu plus important que celui des paramètres jouant sur les bâtiments. De même, l'impact de la proximité reste plus fort que celui de la différence d'altitude.

Ces trois analyses de sensibilité montrent que la proximité, que ce soit à la ligne ou aux gares, joue un rôle important dans la spatialisation de l'élément de localisation "je suis sous une télécabine », au contraire de la différence d'altitude, dont l'impact est plus faible. Un bon paramétrage de la proximité est donc, dans le 
cas de cet élément de localisation, un moyen efficace de réduire la zone de recherche de la victime. L'écart de valeur de l'indice $\mu^{*}$ entre les paramètres utilisés pour la modélisation de l'élément de localisation "je suis sous une ligne de télécabine » et leurs équivalents utilisés pour la modélisation de l'élément de localisation «je suis sous une gare de télécabine » indique une différence d'impact entre la zone construite à partir des gares et de celle construite à partir de la ligne de télécabine. Dans ce cas, la connaissance de la composante de l'objet de référence utilisée par la victime comme objet de référence, serait une indication efficace pour réduire la zone de recherche.

\section{Conclusion}

Dans cet article, nous avons présenté notre démarche visant à construire les zones de présence possible d'un objet dont la position est décrite à partir d'un référentiel spatial indirect à l'aide d'éléments de localisation. Ces derniers ont été modélisés sous la forme de triplets, de manière analogue au modèle de Vasardini et al. (2011). Nous avons cependant étendu ce modèle en y ajoutant le concept de relations spatiales étendues, apportant plus de précision. À partir de ce modèle, nous avons présenté le concept de décomposition des relations spatiales, visant à exploiter les redondances sémantiques des relations spatiales. Nous avons également présenté les problématiques liées à l'incertitude et l'intérêt d'adopter une démarche de modélisation des relations spatiales non-bivalentes, à l'aide de la théorie des ensembles flous. Nous avons ensuite proposé une application comparative de deux implémentations de la théorie des ensembles flous, démontrant l'adéquation de l'approche raster à notre problématique. L'étude d'un second cas réel a mis en évidence l'existence d'objets de références composites, nécessitant une nouvelle étape de décomposition, portant cette fois sur les objets de référence. Enfin, nous avons étudié l'impact des paramètres sur la spatialisation des éléments de localisation.

La décomposition des relations spatiales nécessite un important travail d'analyse, visant à développer une méthodologie d'identification des redondances sémantiques et de l'impact des objets de référence sur la sémantique des relations spatiales, sur la base d'un recensement des relations spatiales et des objets de référence les plus présents dans notre contexte.

Une autre tâche importante est d'automatiser la définition des seuils. Dans les exemples présentés, ces derniers sont définis empiriquement. Cependant, cette approche pose deux problèmes, le premier étant que les seuils sélectionnés ne sont pertinents que pour un type d'objet de référence donné, le second est qu'il serait intéressant de faire varier ces seuils localement pour prendre en compte certaines spécificités du terrain. À titre d'exemple, l'élément de localisation «je suis sous une ligne électrique " nécessite de prendre en compte tout type de ligne et non plus seulement les lignes à haute tension, par conséquent il sera nécessaire de faire varier les seuils précédemment présentés en fonction du type de la ligne électrique (dont dépendent sa hauteur et sa largeur). Le second aspect est que dans un contexte montagneux, la hauteur d'une ligne à haute tension par rapport au sol peut varier 
fortement, au gré des ruptures de pente. On peut postuler qu'il en découle une forte variabilité dans la perception du recouvrement, modélisable par une variation locale des seuils $d_{S}$ et $d_{N}$ (cf. eq. 7). Une des pistes envisagées est de recourir à des méthodes d'apprentissage, dont l'application à l'identification de relations spatiales floues a déjà été expérimentée Xu et al. (2006). Bien que cette approche soit séduisante, la nécessaire constitution d'une base d'apprentissage est un défi de taille compte tenu de l'importance des spécificités locales. Quelle que soit la solution retenue, cette problématique appelle à confronter l'estimation des seuils à une vérité terrain, ce qui nous conduit à envisager l'élaboration d'une enquête de terrain.

Nous souhaitons également aborder la question de l'insertion de la méthodologie présentée au sein du processus de localisation de la victime. Ici, nous avons choisi de calculer directement la zone, mais il serait envisageable d'opter pour une approche itérative, fondée sur des raffinements successifs de la zone estimée. Cette solution, qui peut apporter un gain de performances non négligeable, pose toutefois de nouvelles questions méthodologiques et nous expose au risque de voir apparaître des faux négatifs liés au MAUP (Openshaw, 1984).

À plus long terme, nous souhaitons intégrer la prise en compte de l'incertitude à la méthodologie développée. Comme nous l'avons précédemment expliqué, nous envisageons de traiter l'incertitude (e.g. «je crois que je suis sous une ligne électrique ») lors de l'agrégation des zones correspondant aux éléments de localisation, en vue de construire la zone de présence finale de la victime. La prise en compte de l'incertitude nécessite de coupler la théorie des sous-ensembles flous à une théorie permettant de modéliser l'incertitude telle que la théorie des possibilités ou celle des fonctions de croyances.

Enfin, nous souhaiterions travailler sur le rôle des verbes dans la sémantique d'un élément de localisation. Ces derniers ont étés intégrés dans le modèle, mais ils ne sont pas encore pris en compte dans notre méthodologie de spatialisation des éléments de localisation.

\section{Bibliographie}

Bejaoui L., Pinet Fr., Bedard Y., Schneider M. (2009). Qualified Topological Relation Between Spatial Objects with Possible Vague Shape. International Journal of Geographical Information Science, vol. 23, $\mathrm{n}^{\circ}$ 7, p. 877-921.

Borillo A. (2009). L'espace et son expression en français, Ophrys, Paris.

Bunel M., Olteanu-Raimond A-M., Duchêne C. (2018) Référencement spatial indirect: Modélisation à base de relations et d'objets spatiaux vagues. Spatial Analysis and GEOmatics 2018, SAGEO 2018, Montpellier.

Clementini E. (2008). Objects with Broad Boundaries. Encyclopedia of GIS. New-York, Springer, p. 793-798.

Cohn A.G., Gotts N.M. (1996).The "egg-yolk" representation of regions with intermediate boundary. Geographics Objects With Intermediate Boundary. London, Taylor \& Francis, p. 171-187. 
Objets et relations spatiales composites pour interpréter un référencement spatial indirect. 25

\section{CGAL Project. (2019). CGAL User and Reference Manual.}

De Runz C. (2008). Fouilles archéologiques : à la recherché d'éléments représentatifs. Atelier Fouille de données complexes, Sofia Antipolis.

Dilo A. (2006). Representation of and reasoning with vagueness in spatial information. A system for handling vague objects. Wageningen University.

Dilo A., de By R., Stein A. (2007). A System of Types and Operators for Handling Vague Spatial Objects. International Journal of Geographical Information Science, vol. 21, $\mathrm{n}^{\circ} 4, \mathrm{p}$. 397-426.

Egenhofer M., Mark D. (1995). Naive Geography. International Conference on Spatial Information Theory, Springer, Berlin.

Moncla L., Gaio M. (2015). A Multi-Layer Markup Language for Geospatial Semantic. 9th Workshop on Geographic Information Retrieval 2015, ACM, Paris.

Morris M. (1991). Factorial Sampling Plans for Preliminary Computational Experiments. Technometrics, vol. 33, ${ }^{\circ}$ 2, p. 161-174.

Olteanu-Raimond A.-M., Davoine P.-A., Gaio M., Gouardères E., Van Damme M.-D., Villanova-Olivier M., Brasebin M., Dominguès C., Duchêne C., Favre O., Mustière S., Devin Fl., le Nir Y., Moncla L., Bouveret S., Genoud Ph., Gensel J., Ziebelin D. (2017). Projet CHOUCAS : Intégration de données hétérogènes et raisonnement spatial pour l'aide à la localisation des victimes en montagne. Spatial Analysis and GEOmatics 2017, SAGEO 2017, Rouen.

Openshaw, S., 1984. The Modifiable Areal Unit Problem.

Schneider M. (2008). Fuzzy Spatial Data Types for Spatial Uncertainty Management in Databases. Handbook for Research on Fuzzy Information Processing in Databases. Hershey, Information Science Reference, p. 490-515.

Schneider M. (1999). Uncertainty Management for spatial data in databases: fuzzy spatial data types. Advances in Spatial Databases. Springer, Berlin, p. 330-351.

Tøssebro E., Nygård M. (2002). An advanced discrete model for uncertain spatial data. Lecture Notes in Computer Science. Springer, Berlin, p. 37-51.

Vandeloise C. (1986). L'espace en français. Sémantique des relations spatiales. Éditions du Seuil, Paris.

Vanegas M.-C., Bloch I., Inglada J. (2011). A Fuzzy Definition of the Spatial Relation "Surround". EUSFLAT Conference 2011, Atlantis Press, Aix-les-Bains.

Vasardani M., Timpf S., Winter S., Tomko M. (2011). From Descriptions to Depictions: A Conceptual Framework. Spatial information theory, Springer, Berlin.

Xu J., Yao C. (2006). Formalizing natural-language spatial relations descriptions with fuzzy decision tree algorithm. Geoinformatics 2006.SPIE. Wuhan.

Zadeh L. (1965). Fuzzy sets. Information and Control, vol. 8, n 3, p. 338-353.

Zoghlami A., de Runz C., Herman A. (2016). F-perceptory: an Approach for Handling Fuzziness of Spatiotemporal data in Geographical Databases. International Journal of Spatial, Temporal and Multimedia Information Systems, vol. 1, $\mathrm{n}^{\circ}$ 1, p. 30-62. 